CAPÍTULO 1

\title{
PRODUCTOS DE CONFITERIA Y FERTILIZANTES OBTENIDOS DE FRUTAS Y HORTALIZAS EN EL DEPARTAMENTO DEL ATLÁNTICO
}

\author{
Hilda Helena Estrada-López', Hernán Guillermo Saumett-España², \\ Adriana Marcela Cáceres-Martelo ${ }^{3}$
}

1 Administradora de Empresas, Universidad Autónoma del Caribe. Doctora en Administración de Empresas, Universidad Autónoma de Querétaro, México. Investigadora del Grupo Innovación y Desarrolo Empresarial-GIDE. Universidad Simón Bolívar, Barranquilla (Colombia). Docente en la Facultad de Ciencias Económicas de la Universidad del Atlántico, Barranquilla (Colombia). hileslo@hotmail.co

2 Sociólogo, Universidad Simón Bolívar. Magíster en Proyectos de desarrollo social, Universidad del Norte. Docente-Investigador del Grupo Innovación y Desarrollo Empresarial-GIDE. Universidad Simón Bolívar, Barranquilla (Colombia). herse47t@unisimonbolivar.edu.co

3 Contadora Pública, Universidad Simón Bolívar, Barranquilla (Colombia). acaceres4@unisimonbolivar.edu.co 


\section{RESUMEN}

Este capítulo presenta los principales aspectos del proyecto "Desarrollo de productos de confitería y fertilizantes para la generación de valor agregado asociado a la producción de productos agrícolas en el departamento del Atlántico", seleccionado por Colciencias en la convocatoria 713/2015. La investigación se planteó como objetivo el desarrollo de tecnologías relacionadas con la formulación, evaluación y validación de tres líneas innovadoras de confitería nutracéuticas y una de biofertilizantes, a partir de frutales como mango, guayaba, limón, y hortalizas como el ají, cebollín y ahuyama; productos que responden a las necesidades de las cadenas de producción del sector agropecuario, priorizados en el Plan Nacional de Desarrollo 2014-2018 e incluidas en la agenda de $I+D+i(P N D, 2015)$. Es una forma de agregar valor a productos del sector agropecuario que no realizan transformaciones, aun cuando tienen mercados con el nuevo valor agregado por un proceso de producción. Estos productos nutracéuticos, en la categoría de confitería, se comercializarían de forma directa en tiendas especializadas y bajo la modalidad de licenciamiento. El área de influencia del proyecto comprende el departamento del Atlántico en los municipios de Santa Lucía y Malambo. Los beneficiarios son: La Cooperativa Productora y Comercializadora de Productos Agrícolas del Sur del Atlántico, Cooagrosur, y la Asociación de Fruticultores de Malambo, Asofruma.

Palabras clave: Confitería saludable, biofertilizantes, líneas nutracéuticas, sector agropecuario.

\section{ABSTRACT}

This chapter introduces the main aspects of the project "Development of confectionery products and fertilizers for the genera- 
tion of added value associated with the production of agricultural products in the Department of the Atlantico", which was selected by Colciencias in the call 713/2015. It project, is raised as objective the development of technologies related with the formulation, evaluation and validation of three lines innovative of confectionery nutraceuticals and an of biofertilizers, starting from fruit as mango, guava, lemon and vegetables as the pepper, chives and pumpkin; products that respond to the needs of agricultural production chains prioritized in the National Plan of development 2014-2018 and included in the agenda of $r \& d$ (PND, 2015). It is a way of adding value to agricultural products that do not perform transformations, even when they have markets to consume that product with the new value added by a process of production. These nutraceuticals in the category of confectionery that comercializaria directly in specialist shops and in the form of licensing. The area of influence of the project includes the Department of Atlántico in the municipality of Santa Lucía and Malambo. The beneficiaries of the project are cooperative producer and trading company of agricultural products of the South of the Atlantic, Cooagrosur and the fruit growers Association of Malambo, Asofruma.

Key words: Healthy confectionery, biofertilizers, lines nutracetical, agricultural sector.

\section{INTRODUCCIÓN}

El sector agropecuario tradicionalmente ha tenido una participación importante en el desarrollo económico del país; en el año 1925 representaba el 58,8 \% del Producto Interno Bruto (PIB). Sin embargo, su participación ha ido decreciendo, y para el periodo 1965-1990 el promedio de participación alcanzó tan solo el 23,5 \% del PIB nacional. 
Esta disminución se acentúa en los últimos 24 años, período en el cual el crecimiento del sector agropecuario ha sido muy volátil con respecto al PIB total, cayendo de $9 \%$ a $6 \%$ desde el año 2000 hasta el 2014, con una alta dependencia del café, tal como lo registran los datos aportados por la Misión para la Transformación del Campo (2015).

La problemática del sector agropecuario está asociada a múltiples causas, entre ellas la focalización de las inversiones públicas en grandes productores, dejando de lado a los pequeños que representan un componente importante del total. Otra de las causas es la incompleta apertura económica realizada en la década del 90 que tuvo fuerte impacto en la reducción del área sembrada, situación que se empezó a restablecer hasta 1997 y 1998 (Misión para la Transformación del Campo, 2015).

La causa que originó este proyecto es la poca oferta exportable, asociada a la escasa transformación y generación de valor de productos relacionados con frutas y hortalizas.

El departamento del Atlántico tiene una extensión de $3.386 \mathrm{Km} 2$, con un área que representa el 0,29\% de la extensión total del país (Gobernación del Atlántico, 2014). El Producto Interno Bruto (PIB) del departamento del Atlántico en el año 2012 fue de $\$ 25.078$ miles de millones, lo que correspondió a un 3,8 \% del PIB nacional (DANE, Banco de la República, 2013), siendo el departamento en su mayor proporción un territorio rural; el aporte del sector agropecuario fue de 1,9 \% del PIB departamental en el año 2012; el aporte del subcomponente frutas, legumbres, hortalizas, aceites y grasas procesadas fue de tan solo 0,63\% en el período 2000-2009 (Departamento del Atlántico, 2012). 
El sector agrícola del Departamento registra un deterioro en cuanto a su participación en el PIB departamental, situación que se acrecentó luego de los efectos de la ola invernal de los años 2010 y 2011. Cifras del año 2013 aportadas por el DANE, Banco de la República (2013) muestran que la actividad agrícola está concentrada en cuatro cultivos permanentes: yuca $(59,11 \%)$, limón (13,15 \%), mango $(9,41 \%)$, guayaba $(2,69 \%)$. En cuanto a los cultivos transitorios, el Departamento produce maíz (29,04 \%), melón (17,07\%), patilla $(12,34 \%)$, ahuyama $(12,18 \%)$ y ají $(2,99 \%)$ La producción agrícola del departamento del Atlántico durante el 2013, en lo referente a los productos que tendrán un aprovechamiento en el desarrollo del proyecto, son: limón, mango, guayaba, naranja, ahuyama y ají.

En lo que respecta a la problemática del componente agroindustrial, Nucci y Morales (2009) señalaron que el sector hortofrutícola se ha manejado, en gran medida, de forma empírica y con escasa incorporación de tecnología asociada a factores como:

- Ausencia de cultura poscosecha para conservar y/o transformar las frutas y hortalizas generando valor.

- Escasa tecnificación de cultivos, lo que se traduce en calidad irregular, generando barreras para la comercialización en mercados internacionales.

- Escaso desarrollo de la asociatividad entre pequeños productores, transformadores y comercializadores.

La transformación de frutas en el departamento del Atlántico es liderada por la Compañía Envasadora del Atlántico (CEA, 2015), fundada en el año de 1982 y que actualmente exporta a 39 países 
sus cinco líneas de productos. Sin embargo, los pequeños productores de frutas y hortalizas del Atlántico, no tienen acceso a comercializar sus productos por este canal y requieren de alternativas para generar valor a su producción.

Los municipios de Santa Lucía y Malambo, área de influencia del proyecto, tienen una marcada vocación agropecuaria y, por ello, la Universidad Simón Bolívar desde hace seis años viene trabajando en estos municipios en el desarrollo de proyectos de investigación, con el propósito de generar soluciones a la problemática asociada a la baja productividad del sector agrícola. Los productores de frutas y hortalizas, ubicados en estos dos municipios, no le están generando valor agregado a la producción primaria, y en los períodos de cosecha se desaprovecha el exceso de producción hasta el punto de perder productos ante el bajo nivel de precios. Por otra parte, la falta de oportunidades da como resultado la emigración de la población joven a otros municipios o ciudades en busca de mejores opciones de vida, y cada vez es más frecuente el aumento de la población adulta campesina vulnerable y los altos índices de desnutrición de la comunidad en general. En el municipio de Santa Lucía (Sánchez, 2011) el desempleo corresponde a cifras superiores al 60 \% después de la inundación y pérdida de cultivos en la zona. En la actualidad se han venido generando aportes a los damnificados para siembras de productos de la zona, como son guayaba y mango, entre otros.

\section{METODOLOGÍA}

El área de influencia del proyecto comprende el departamento del Atlántico en los municipios de Santa Lucía y Malambo. Para el desarrollo de los productos se utilizarán las materias primas existentes 
en las zonas; (mango, guayaba, limón, ahuyama, ají y cebollín) inicialmente se determinarán las calidades y cantidades de producción, los tiempos de cosecha y la variabilidad de especies de cada uno de los alimentos, con el fin de programar las actividades con los participantes en el proyecto.

Con relación a la metodología planteada, Hernández (1991), se refieren a las estrategias que se desarrollarán para llevar a cabo la investigación. En el siguiente diseño metodológico se ilustra cada una de las fases del proyecto con base en los objetivos planteados.

Fase 1. Formulación, pruebas y estandarización de nuevos productos: los objetivos 1 y 2 , se abordarán bajo un estudio descriptivo, experimental y cuantitativo, en el cual, a partir de un problema de pérdida de materias primas, se hace énfasis en la transformación de productos como alternativa de solución y valor agregado para las poblaciones de Santa Lucía y Malambo. Descriptiva porque busca especificar las propiedades, características y los perfiles de personas, grupos, comunidades o cualquier otro fenómeno que se someta al análisis. La experimental (Muggenburg y Pérez, 2007) se caracteriza por la intervención intencionada y programada en la que se manipulan una o más variables, con el fin de analizar las consecuencias que la manipulación ejerce sobre las variables dependientes y sus efectos. La cuantitativa (Monje, 2011) se caracteriza por ser un proceso sistemático y ordenado que consiste en proyectar el trabajo de acuerdo con una estructura lógica de decisiones y estrategias para la obtención de respuestas adecuadas a los problemas de indagación propuestos en el estudio. El diseño de investigación es una estructura o un plano que sirve para dirigir un proyecto de investigación, detalla los pasos necesarios 
para obtener la información indispensable en el problema que nos ocupa, para este documento, la utilización de las materias primas como mango, ahuyama, guayaba, limón, ají y cebollín, y la utilización de los residuos de cosecha y producción con el fin de elaborar un biofertilizante que cuide el medioambiente.

Se requiere de un diseño (Hernández Sampieri, 2003) estrategia que se desarrolla para obtener la información que se requiere para la investigación, especificando los detalles y asegurando que el proyecto se realizará de manera efectiva y eficiente. En el diseño se incluyen los siguientes pasos: 1. Definir la información necesaria para la caracterización de materias primas; 2 . Diseñar las fases descriptivas por cada etapa del proceso; 3. Especificar los procedimientos para medir y elaborar escalas, relacionadas con el muestreo estadístico y sus respectivos análisis; 4. Construir y probar formas apropiadas de recolección de datos para establecer los productos con mayor aceptabilidad por los consumidores en las diferentes veredas; 5 . Especificar los procesos de muestreo y el tamaño de la muestra, y 6. Desarrollar un plan para el análisis de los datos recolectados, seleccionando los productos más aceptados para ser estandarizados.

Fase 2. Evaluación económico-financiera, mercado y organizacional: la evaluación de los aspectos económico-financieros, de mercado y organizacional, asociados al desarrollo de las nuevas líneas de confitería nutracéutica que contribuyan al desarrollo empresarial de los pequeños productores agrícolas, parte de la revisión del estado del arte en lo referente a publicaciones sobre evaluación de componentes organizacionales, de mercado, y económicos-financieros de procesos similares, para tener referentes 
teóricos que enmarcarán la ruta más apropiada en el desarrollo de este trabajo.

\section{Población y Muestra}

Tamayo (2008) entiende por población a la totalidad del fenómeno a estudiar, en donde las unidades de la población tienen características comunes susceptibles de observación, hecho que da origen a los datos de la investigación. La población de este estudio estará representada por los propietarios de las fincas, o unidades productivas del sector agrícola, en los municipios de Santa Lucía y Malambo del departamento del Atlántico, y por los usuarios finales participantes del proyecto y que están asociados a las cooperativas agrícolas.

La evaluación del mercado comprende tres estudios, uno dirigido al segmento de distribuidores (tiendas, graneros y supermercados), para evaluar la satisfacción que tienen con los proveedores actuales de la línea de confitería y las expectativas alrededor de la categoría; otro dirigido al consumidor final para analizar la satisfacción con la oferta actual de líneas de confitería, y las expectativas alrededor de la categoría, una prueba de mercado de los productos y diseño de estrategias.

Teniendo en cuenta lo anterior, para la evaluación de mercado se utilizó un método de corte cuantitativo. Como el universo corresponde a una población infinita, es decir, mayor a 30.000.000, la muestra para la población, de acuerdo a lo que plantea González (2008) para estos casos, se expresa de la siguiente manera: $\mathrm{N}=\left(Z_{-} c^{\wedge} 2 ? p ? q\right) / \mathrm{e}^{\wedge} 2$. 
Donde:

$\mathrm{N}=$ tamaño de la muestra.

Z_c^2 = valor que determina el nivel de confianza.

$p=$ proporción de los elementos que determinan la característica de la investigación.

$q$ = proporción de los elementos que no determinan la caracte rística de la investigación.

El análisis de los datos cuantitativos se realizó usando el programa SPSS.

El segmento de los distribuidores estará conformado por tres subgrupos: tiendas, graneros y supermercados. La persona a encuestar es el propietario o administrador, si se trata de tiendas de barrio y panaderías; para los supermercados de cadena, quien se encargue de las compras de frutas.

La muestra del total de tiendas equivale a $94 \%$ de nivel de confianza y $2 \%$ de error muestral con muestreo simple aleatorio para proporciones. El trabajo de campo para el segmento de los distribuidores se obtiene con un cuestionario que se aplicó personalmente y sin cita, garantizando que la persona cumpla el perfil; se cubrieron diez barrios en diferentes zonas de los estratos 2 a 5. Las tiendas deberán ser medianas o grandes; se excluyen las pequeñas.

El grupo objetivo de los consumidores finales de líneas de confitería; serán personas que respondan por la mayoría de compras de su hogar. Pueden ser hombres o mujeres, residentes en estratos 2 a 5. El número de encuesta a aplicar en este segmento corresponde 
a $95 \%$ de nivel de confianza y $5 \%$ de error muestral con muestreo aleatorio simple, número muy utilizado para este tipo de estudios.

El Estudio Económico Financiero está asociado a todo tipo de actividades económicas y organizaciones empresariales (Del Río, 2007). Por otra parte, Cuevas (2001) señala que la contabilidad de costos desempeña un papel destacado en los informes financieros, son un elemento fundamental en la determinación del ingreso $y_{\text {, }}$ por supuesto, de las utilidades o pérdidas en una actividad económica o en un proyecto.

Para la realización de la evaluación económica se seleccionó el sistema de costeo por procesos, teniendo en cuenta la naturaleza del proyecto donde el modelo de producción se sustenta en procesos y subprocesos. El sistema de costeo por procesos es adecuado para actividades económicas con características continuas (Cuevas, 2001).

El análisis de los procesos organizacionales estará dirigido a las empresas cooperativas que asocian a los pequeños productores agrícolas. El método que se utilizará para este estudio será el deductivo, ya que se tomarán elementos directamente de los pequeños productores agrícolas, para proceder a deducir cuál es la forma y dirección que asume la organización en estas pequeñas unidades productivas asociadas y las cooperativas.

Se utilizará la metodología cualitativa, específicamente la técnica de entrevista en profundidad, con el ánimo de analizar en un ambiente propicio las percepciones de los pequeños productores agrícolas sin que haya otras influencias. Como indica Weirs (2006), 
la técnica hace que el moderador aliente al entrevistado para que exprese con entera libertad sus ideas sobre el tema de estudio y, en ciertos casos, se convierte en una ventaja sobre la sesión de grupo, pues la persona puede expresar inquietudes que no manifestaría en público. Se eligió la metodología cualitativa porque se pretende conocer aspectos psicológicos de la forma como realizan la administración de sus fincas.

Con la entrevista se espera reconstruir los elementos que conforman los perfiles socioeconómicos de cada unidad de estudio y de su propietario, ubicados en la zona urbana y en el área rural de los municipios de Santa Lucía y Malambo del departamento del Atlántico. Asímismo, la entrevista reflejará la situación de los pequeños productores agrícolas. Se espera tener información importante para conocer la forma en que ellos han aplicado en sus unidades productivas conceptos de administración; aquí se puede captar cuál es la capacidad con que cuentan, cómo gestionan la calidad de sus productos, los aspectos más significativos para la marcha del negocio, y la estrategia que emplean para garantizar el crecimiento del negocio y la manera de verificar los resultados obtenidos. Para fortalecer el estudio se utilizarán herramientas cuantitativas.

\section{ESTADO DEL ARTE}

\section{Productos de confitería}

Los resultados de la Encuesta Nacional Agropecuaria (ENA Atlántico 2012), establecen para el uso del suelo agrícola un total de 13.623 hectáreas, pecuaria de 244.241 hectáreas, bosques 8.642 hectáreas, y otros usos 11.013 hectáreas. El área sembrada de frutales en el departamento del Atlántico es de 65.135 ha, que corresponde a un porcentaje nacional de 0,9\%. Según el Plan Frutícola 
Nacional (2006), las pérdidas poscosecha se deben al mal manejo que dificulta la comercialización. El deterioro también es producido por causas mecánicas, como empaque inapropiado, transporte, lo que provoca golpes dando lugar a magulladuras, manchas, ablandamiento, que genera la mala calidad de los frutales. El perfil económico del departamento del Atlántico realizado por el Ministerio de Comercio, industria y turismo, en el 2013, establece la producción en porcentajes de cultivos transitorios como ahuyama (12,18\%), cebolla en rama (1,0 \%), maíz (29,04%), melón (17,07%), ají (2,99\%) entre otros productos, y para cultivos permanentes, como mango $(9,41 \%)$, guayaba $(2,69 \%)$, limón (13,15\%).

Entre las alternativas de transformación agrícolas actuales para el departamento el Atlántico, según el Ministerio de Agricultura (2015), son importantes productos con altos contenidos nutricionales, que permitan procesos de producción con valores agregados y posibilidades de exportación. Entre las alternativas de transformación se encuentran alimentos como la guayaba, ahuyama, mango, maíz, yuca, de acuerdo con el concepto de Hernández, R. (2015) del programa de cadenas productivas del Ministerio. La fortificación de alimentos es cada vez más popular en los países en vías de desarrollo. Un ejemplo es el documento realizado por Rocha y Coy (2006) sobre bebidas fortificadas a base de leche de soya, y ahuyama con hierro y calcio para adultos mayores. Las combinaciones de harinas con ahuyama (Velasco, 2015) para la elaboración de cakes y tortas, son otro uso en la industria confitera colombiana, aprovechando los componentes nutricionales de la ahuyama y sus características organolépticas y sensoriales. Otros productos de confitería (Molina, 2014) como chocolates rellenos con crema de mango y ají en salsa de mango, han sido premiados por ser 
productos innovadores de la Universidad Técnica de Manabí en el festival del mango. El sector hortícola (2013) ha solicitado un plan de negocios al gobierno nacional para priorizar siete productos, para recibir apoyo del programa de transformación productivo PTP. Los productos son: papaya, piña, mango, fresa, aguacate, cebolla de bulbo y ají, utilizados en procesos de confitería por estudiantes del Sena con sede en Guadalajara de Buga, Valle del Cauca. En ambientes empresariales como Ciproba La Costiera, 2015), se han realizado productos de innovación en frutales como diferentes tipos de vinagretas, entre las cuales se encuentran: trozos de mango y toque de cilantro, base de piña o uchuva con vinagre y combinación de ají o pimienta con el limón.

La Costiera (2015) ofrece varios tipos de confitería, como los daba al limoncillo, un licor a base de limón para productos de confitería especialmente. La confitería funcional Nexira (2015) la define como aquel producto al cual se le ha agregado un ingrediente que actúa sobre componentes funcionales específicos corporales, pero además es nutritivo. Los mercados globales de productos funcionales para confitería han crecido 38 \% desde el año 2006, y al 2014 se tiene un mercado de $U \$ 11.000$ millones; sin embargo, se considera que estos productos siguen siendo nichos y todavía pueden pasar a cadenas de mercadeo mayores.

Scientific Psychic demuestra que la sucralosa se promueve como un edulcorante sin calorías, pero en realidad es una mezcla de dextrosa, malto dextrina, y sucralosa. Diez gramos de Splenda contienen $9.00 \mathrm{~g}$ de carbohidratos que incluyen $8.03 \mathrm{~g}$ de azúcares (dextrosa) y 0,96 gramos de almidón (malto dextrina). Por esta razón, 10 gramos de Splenda tienen 33 kilocalorías comparados con 39 
kilocalorías en un peso igual de azúcar. Las calorías de Splenda provienen de los carbohidratos, y no de la sucralosa.

Formulaciones recientes de Splenda utilizan maltodextrina resistente, que se puede categorizar como fibra. La confitería o tecnología de los productos dulces es un área de la tecnología de alimentos, dividida en dos ramas: 1. El confite corresponde a productos como el caramelo duro, suave, caramelo aireado, pastillas de goma y todo tipo de chocolates; 2 . El confitado corresponde a productos con recubrimientos de dulce como frutas, centros dulces duros y suaves, gomas de mascar, centros de chocolate con semillas.

Las tendencias de los alimentos nutracéuticos, de acuerdo con IPF (2015), tienen gran demanda por los beneficios que dan para la salud. El mercado está creciendo rápidamente, con un valor pronosticado para 2016 de U\$207 millones, debido a que cada día los consumidores buscan alimentos saludables, multi-tarea, productos alimenticios y bebidas. Cada día se investiga sobre diferentes componentes para productos de confitería (IPF, 2015), como es el caso de la goma de acacia, que no se pega a los dientes y mejora la liberación de aromas y sabores con mayores lapsos de tiempo, además de otros componentes naturales para este tipo de productos.

El mercado de los productos horneados y cereales es de casi el $23 \%$ del mercado mundial de alimentos funcionales, con ventas por valor de $U \$ 5,5$ mil millones entre los años 2006-2010, mostrando un crecimiento anual del $28 \%$. En cuanto a los productos de panadería y cereales enriquecidos con fibra, minerales y vitaminas, aumentaron el valor del sector en casi un $54 \%$ en los últimos años.

El Instituto de Innovación en Biotecnología e Industria, IIBI (Food 
NewsLatam, 2014), en República Dominicana, ha realizado una transferencia de tecnología a las microempresas de panificación, con la elaboración de panes y bizcochos (utilizando harinas de ahuyama, yuca, plátano y batata). Con las nuevas alternativas se puede sustituir entre el $10 \%$ al $50 \%$ de harina de trigo por una o varias harinas en la elaboración de productos alimenticios.

La industria confitera divide los dulces en tres clases: los elaborados con chocolate, los de repostería y pastelería, y los dulces con base en azúcar. Los principios básicos para la producción de confites son la realización de un balance de materia para la formulación, la preparación y mezcla de los ingredientes, concentración de mezcla hasta temperaturas deseadas, enfriamiento, moldeo, empaque y rotulado. Entre los factores que afectan la producción y almacenamiento de los dulces se encuentra: el grado de inversión del azúcar, el tiempo, la temperatura de concentración, la humedad relativa, la humedad residual del producto y otros ingredientes.

La preparación de formulaciones basadas en concentraciones mayores al $65 \%$ resulta en un incremento del módulo de corte debido a la reducción de la movilidad de biopolímeros y a la conformación de la red estructural del producto (Kasapis et. al., 2004). A pesar de las investigaciones realizadas no se han encontrado estudios que reporten la elaboración de un dulce duro o blando a partir de pulpas de fruta, legumbres y tubérculos, adicionados con vitaminas de manera natural y no incorporadas artificialmente.

En la actualidad se encuentra una oferta importante de alimentos más "sanos" desarrollados por la industria alimentaria, encontrando alimentos de diseño, que son suplementados con ingredientes 
naturales ricos en sustancias capaces de prevenir enfermedades, como por ejemplo: fotoquímicos (sustancias que se encuentran en verduras y frutas), fibra dietaria, ácidos grasos poliinsaturados (omega 3) y probióticos (bacterias vivas aportadas por alimentos fermentados), pero aún no se cuenta con una definición de alimentos funcionales y nutracéuticos. Sin embargo, el Dr. Stephen De Felice, presidente de la Fundación para la Innovación en Medicina, definió la palabra nutracéuticos como "un alimento o parte de un alimento que proporciona beneficios médicos o para la salud, incluyendo la prevención y/o el tratamiento de enfermedades" 2015, p.1.

Los productos nutracéuticos son alimentos de origen natural con propiedades biológicas activas que, aparte de nutrir, aportan beneficios para la salud. Estos alimentos deben ser transformados por métodos no desnaturalizantes, que aporten estabilidad y que sean seguros para el consumo de la población en general.

Biofertilizantes: según Brechelt (2004), la nueva tendencia de medioambiente libre de contaminantes, ha desarrollado la creación y formulación de los biofertilizantes a partir de desechos de cosechas y de procesos de producción; están definidos como productos a base de microorganismos benéficos del suelo, con uno o varios microorganismos que viven asociados o en simbiosis con las plantas y ayudan de manera natural a su nutrición y crecimiento, pero además son mejoradores del suelo.

Para Acuña (2012), las ventajas del uso de biofertilizantes permiten una producción a bajos costos, protección del medioambiente y la conservación del suelo para que mantenga la fertilidad y biodiversidad. La formulación se desarrolla teniendo en cuenta, como 
fuentes de nitrógeno, residuos de cosecha, desechos animales, nitrógeno atmosférico, lluvias y microorganismos. Las simbiosis más conocidas están relacionadas por: leguminosas-rhizobium, plantas actinorrizicas y frankis, helecho azolla y anabaena, liquen y cianobacterias. Iglesias (2010) informa que existe la Red Biota, conformada por 53 grupos de investigadores pertenecientes a once países, entre esos algunos latinoamericanos, que trabajan en el desarrollo de biofertilizantes. Desde el punto de vista social y político, pueden contribuir con la equidad mundial; su producción es relativamente barata y es posible producirlos en cualquier lugar del mundo.

Los biofertilizantes son productos altamente tecnológicos y en la actualidad su producción no está al alcance de todos. Consta de un principio activo, que es el microorganismo elegido y el inoculante, cuya formulación determina en gran parte su eficiencia. Los fertilizantes mixtos (Abreu y Ferreira, 2014) se toman de bases de microorganismos que viven en el suelo y en las plantas, y que cumplen funciones directas o indirectas en la nutrición, logrando la biodisponibilidad de elementos esenciales para el suelo y el mejoramiento de los cultivos.

\section{RESULTADOS PARCIALES}

El proyecto en la actualidad se encuentra en la etapa de ejecución para el cumplimiento de los objetivos y establecer las condiciones óptimas, tanto en materias primas en fresco como de los equipos, utensilios y demás materiales requeridos en el desarrollo del proyecto. Pero, además de los estudios de mercadeo, establecer los alimentos de mayor aceptación por los consumidores, se tomará como base esta información para desarrollar las formulaciones co- 
rrespondientes de los alimentos que serán presentados, de suerte que los productos con mayores porcentajes de aprobación por la población serán estandarización, y su formulación se les entregará a las dos cooperativas con sus respectivas fichas técnicas y registros sanitarios.

La visita a las diferentes veredas de los municipios de Santa Lucía y Malambo permitió establecer las calidades de las materias primas, realizar las pruebas de plataforma iniciales y determinar las cantidades de frutas y hortalizas que se cosechan en la zona. Es decir, a partir de los análisis de laboratorio de las materias primas de mango, verde y limón amarillo, se establecieron las pruebas de plataforma iníciales requeridas para las formulaciones, dando como resultado buena calidad microbiológica, encontrándose dentro de los valores de referencia normal.

Con el objetivo de garantizar la participación de los beneficiarios en la etapa de formulación de las tres líneas de confitería nutracéutica, chocolatería, panadería y repostería, se desarrollaron capacitaciones de manipulación de alimentos para las líneas de producción, y manejo de los instrumentos requeridos para la toma de información sobre diámetros internos, externos y profundidad (calibrador o pie de rey), toma de densidad (picnómetro), toma de acidez y basicidad (pH-Meter), toma de grados brix (refractómetro), determinación del grado de madurez de las frutas y vegetales (tinción con lugol al $1 \%$ ), toma de pesos de los diferentes frutos (balanza), toma de temperatura (termómetro), toma de tiempos de actividades (cronómetro), con el fin de que la población comprenda la terminología, necesaria para el logro de los objetivos del proyecto.

Además, se indicaron las características de análisis de calidad física 
para las frutas y vegetales, como aspectos generales, defectos físicos, deshidratación, defectos fisiológicos, defectos biológicos, defectos mecánicos, con el fin de establecer la calidad requerida para las materias primas para el estudio.

En la misma actividad se presentaron las instrucciones para el manejo de materias primas con el fin de iniciar los procesos de deshidratación y obtención de harinas de mango, guayaba, y ahuyama, requeridos para el desarrollo de los diferentes tipos de formulaciones para chocolatería, panadería y repostería.

En la evaluación del mercado se realizaron encuestas, estas se encuentran en estudio para analizar y determinar las expectativas que tiene el consumidor final en cuanto a las tres líneas de confitería.

\section{REFERENCIAS BILIOGRÁFICAS}

Abreu, M. Y. J. y Ferreira C. J. J. (2014). Biofertilizante mixto a base de bacterias de vida libre fijadores de nitrógeno y solubilizadoras de fósforo. MPPAT. Instituto de Salud Agrícola Integral INSAI. Laboratorio Comunal de Producción de Biofertilizantes "Bolívar Conservacionista" Venezuela, Calabozo Guaríco.

Acuña, N. O. (2012). Laboratorio de bioquímica de procesos orgánicos. Centro de investigaciones agronómicas. http://cep.unep. org/repcar/capacitacionyconcienciacion/cenat/biofertilizantes. pdf

Agroindustria Hortícola (2013). Otras Noticias. Boletín Noviembre. https://www.ptp.com.co/contenido/contenido_imprimir.aspx? conID $=530$ \& cat ID $=643$

Brechelt, A. (2004). El manejo ecológico de plagas y enfermedades. Red de acción de plaguicidas y sus alternativas para 
América Latina (RAPAL). http://www.rapal.org/articulos_files/ Manejo_Ecologico_de_Plagas_A.Bretchel.pdf

CEA (2015). Compañía Envasadora del Atlántico. Obtenido de http://www.cea.net.co/index.php?option=com_content\&view $=$ article\&id $=1 \&$ ltemid=148\&lang $=e s$

Ciproba (2015). Salsas y vinagretas de frutas. http://ciproba.com/ productos/salsasdefruta

Cuevas, C. (2001). Contabilidad de costos: enfoque gerencial y de gestión. 2da. Edición. Bogotá, D. C. Pearson Educación de Colombia LTDA.

DANE - Banco de la República (2013). Informe de Coyuntura Económica Regional Atlántico-ICER 2013. Bogotá D.C.: DANE Banco de la República.

Danhke, L. (1989). Metodología de la Investigación. http://catarina. udlap.mx/u_dl_a/tales/documentos/lad/arenas_m_a/capitulo3. pdf

De Felice, S. (2015). Sociedad española de nutracéutica médica. http://www.nutraceuticamedica.org/definicion.htm

Del Río, C. (2007). Generalidades de Costos. Contabilidad de Costos. México: Editorial Ecafsa. http://www.lafacu.com/ apuntes/contabilidad/Costos_l/default.ht

Departamento del Atlántico (2012). Plan de Desarrollo Atlántico 2012-2015 "Atlántico más Social". Barranquilla D.E.I.P.: Departamento del Atlántico.

Encuesta Nacional Agropecuaria en Atlántico (2012). http://www. dane.gov.co/files/investigaciones/agropecuario/enda/ena/Presentacion_Atlantico_2012.pdf

Food New Latam (2014). Elaboración de harinas de ahuyama, yuca, plátano y batata en Rep. Dominicana. Septiembre. http:// www.foodnewslatam.com/biotecnolog\%C3\%ADa/59ingredien- 
tes/100elaborar\%C3\%A1 nharinasdeauyama,yucapl\%C3\%A1tanoybatataenrepdominicana.html

Gobernación del Atlántico (2014). Presentación del Departamento. Disponible en: http://www.atlantico.gov.co/index.php/ presentaciondepartamento

González, R. (2008). Aspectos básicos del estudio de muestra y población para la elaboración de los proyectos de investigación. http://ri.bib.udo.edu.ve/bitstream/123456789/615/1/TESIS_ RGyFS--\%5B00600\%5D--(tc).pdf

Hernández, R. (2015). Metodología de la Investigación. http://catarina.udlap.mx/u_dl_a/tales/documentos/lad/arenas_m_a/capitulo3.pdf

Iglesias, M. (2010). Biofertilizantes, Tesoro desconocido. http://innovacionchile.blogspot.com/2013/08/agriculturabloggerbiofertilizantes.html

Ingredientes y Productos Funcionales IPF (2015). La funcionalidad en los alimentos. http://www.ipf.com.co/es/?option=com_content\&view=article\&id=352: nuevosinnovadoresconfuturo\&catid=53:junio2012\&ltemid=134\&lang=es

Kasapis, S., Mitchell, J., Abeysekera, R., MacNaughtan, W. (2004). Rubberto-glass transitions in high sugar/biopolymer mixtures. Trends in Food Science \& Technology, 15, 298-304.

La Costiera (2015). Limón de Sorrento igp. http://www.la-costiera. com/es/nuestros-productos/limon-de-sorrento-igp.html

Malhotra, N. (1997). Metodología de la Investigación. http://catarina.udlap.mx/u_dl_a/tales/documentos/lad/arenas_m_a/capitulo3.pdf

Méndez, C. (1995). Metodología, Diseño y Desarrollo del proceso de Investigación. Bogotá, Colombia: Editorial McGraw-Hill.

Ministerio de Agricultura (2015). Anuario Agropecuario. Actualiza- 
ción. Formato automatizado perfil de departamento-Ministerio. www.mincit.gov.co/descargar.php?id=71224

Ministerio de Agricultura (2015). Cadenas productivas. Ing. Néstor Julio Hernández. Agroexpo.

Misión para la Transformación del Campo (2015). Departamento Nacional de Planeación. https://colaboracion.dnp.gov.co/CDT/ Prensa/DOCUMENTO\%20MARCO-MISION.pdf

Molina, T. (2014). Ají y chocolate con mango. Conversatorio para el quinto festival de mango de la carrera de Ingeniería Química. Universidad Técnica de Manabí. http://www.utm.edu.ec/seguimosavanzando/index.php/ajiychocolateconmango/

Monje, C. A. (2011). Metodología de la investigación cuantitativa y cualitativa. Guía didáctica. Neiva: Universidad Sur Colombiana, Facultad de Ciencias Sociales y Humanas.

Muggenburg, M. C., Pérez, I. (2007). Tipos de enfoque de investigación cuantitativa. Revista de Enfermería universitaria ENEOUNAM, 1(4), 36-38.

Nexira. Confitería. http://www.nexira.com/es/Confiteria_269.htm

Nucci, S. y Morales, L. (2009). Presente y perspectivas de la agroindustria frutícola exportadora en el departamento del Atlántico. Desarrollo Gerencial, (1), 14-27. Ediciones Universidad Simón Bolívar.

Plan Frutícola Nacional (2006). http://www.asohofrucol.com.co/ archivos/biblioteca/biblioteca_98_Plan\%20Nal\%20fruratlantico. Pdf

Plan Nacional de Desarrollo PND 2015-2018. Todos por un nuevo país. http://wp.presidencia.gov.co/sitios/especiales/Documents/20150616-especial-plan-nacional-desarrollo/index. html\#content

Rocha, R., Coy, S. (2006). Elaboración de una Bebida a Base de Leche 
de Soya y Ahuyama Fortificada con Hierro y Calcio, para Adultos Mayores. Trabajo de grado. Universidad de la Salle http://repository.lasalle.edu.co/bitstream/handle/10185/15582/00798447. pdf?sequence $=1$

Sánchez, A. (2011). Documento de trabajo sobre economía regional. Banco de la República.

Tamayo, M. (2008). Metodología formal de la investigación científica. Bogotá: Limusa.

Velasco, H. (2015). Cakes y tortas de ahuyama. Escuela Gastronómica de Occidente.

Weirs, R. (2006). Investigación de Mercados. México: Prentice Hall. 79.

Zamora, A. (2015). Edulcorantes y azúcares artificiales. Estructura química. Scientific Physic. http://www.scientificpsychic.com/ fitness/edulcorantes-artificiales.html

\footnotetext{
Cómo citar este capítulo

Estrada-López, H. H., Saumett-España, H. G., \& Cáceres-Martelo, A. (2017). Productos de confitería y fertilizantes obtenidos de frutas y hortalizas en el departamento del Atlántico. En H. H. Estrada-López, H. G. Saumett-España, M. A. Iglesias-Navas, M. J. Bahamón, A. M. Cáceres-Martelo, C. E. Restrepo Flórez, . . A. Díaz Pérez, Productos de confitería nutracéutica. Una opción empresarial para cultivadores de frutas y hortalizas (pp.17-40). Barranquilla: Universidad Simón Bolívar.
} 